Article

\title{
Feasibility Evaluation of CFD Approach for Inhalation Exposure Assessment: Case Study for Biocide Spray
}

\author{
Donggeun Park ${ }^{1}$ and Jong-Hyeon Lee ${ }^{2 *}$ \\ 1 Department of Advanced Materials and Parts of Transportation Systems, Pusan National University, 2, \\ Busandaehak-ro 63beon-gil, Geumjeong-gu, Busan, Rep. of Korea; dgpark94@pusan.ac.kr \\ 2 Research Institute of Environmental Health and Safety, 410, Jeongseojin-ro, Seo-gu, Incheon, Rep. of Korea \\ 2; jhleecheju@gmail.com \\ * Correspondence: jhleecheju@gmail.com; Tel.: +82-32-677-0853
}

Featured Application: In the field of exposure science, approach for exposure evaluation using CFD was found that complements the shortcomings of the conventional methodology, the zerodimensional spray model and measurement method.

\begin{abstract}
Consumer products contain the chemical substances that threaten human health. The zero dimensional modeling methods and experimental methods have been used to estimate the inhalation exposure concentration by the consumer products. The model and measurement methods have the spatial property problem and time/cost consuming problem, respectively. For solving the problems due to the conventional methodology, this study investigated the feasibility of applying CFD for evaluation of inhalation exposure by comparing the experiment results and the zero-dimensional results with CFD results. To calculate the aerosol concentration, the CFD was performed by combined the 3D Reynolds averaged Navier Stoke's equation and discrete phased model using ANSYS FLUENT. As a result of comparing the three methodologies performed under the same simulation/experimental conditions, we found the zero-dimensional spray model shows approximately 5 times underestimated inhalation exposure concentration when compared with the CFD results and measurement results in near field. Also, the results of the measured concentration of aerosols at five locations and the CFD results at the same location were compared to show the possibility of evaluating inhalation exposure at various locations using CFD instead of experimental method. The CFD results according to measurement positions can predict rationally the measurement results with low error. In conclusion, in the field of exposure science, a guideline for exposure evaluation using CFD was found that complements the shortcomings of the conventional methodology, the zero-dimensional spray model and measurement method.
\end{abstract}

Keywords: inhalation exposure assessment; computational fluid dynamics (CFD); biocides; spray model; unsteady RANS; ConsExpo;

\section{Introduction}

Consumer products such as the biocide spray have a variety of chemical substances, which intimidate the human health. The humans are exposed to the threaten substance released in daily life. The potential risks of consumer products should be assessed to ensure the human safe. The modeling methods [1-6] and experimental methods $[7,8]$ have been used to estimate the inhalation exposure concentration by the consumer products.

The modeling tools are the mathematical model of ordinary differential equation that can quickly estimate the inhalation exposure from use of consumer products such as the biocide spray, cosmetics and cleaning products. The modeling tools assume that the aerosol particles spread rapidly 
throughout the space, so the air concentration results calculated by the tools is uniform throughout the space [1-4]. The modeling tools cannot account for the inhalation exposure by considering spatial property and hence no obtain the exposure information on the various spots in room. So, the model was called as the zero-dimensional spray model.

Unlike the zero dimensional model's assumption, [9] showed that the concentration of pollutants is not uniform throughout the indoor because the mean age of air varies depending on the measurement locations of the indoor. Also, the exposure experimental results according to the measurement locations were up to 10 times higher than the results of zero dimensional spray model [4]. In addition, a spatially divided multi-box zero-dimensional model has been developed, but the dynamic behavior of aerosols is still unpredictable by the instantaneous diffusion assumption [10]. The zero dimensional spray models are likely to underestimate for the inhalation exposure. The model should consider the spatial property to predict the correct inhalation exposure under the inhomogeneous concentration in indoor.

For solving the spatial problem of the exposure modeling tool, the exposure assessment has to be proceed by experimental method, which can consider the exposure results by the various measurement position and the local ventilation rate by the difference room structure and size. However, experimenting in various measurement locations consumes a lot of equipment cost. Also, the execution time about the experimental analysis can be in the order of weeks and months for the exposure assessment according to the various indoor space specifications. That is, an appropriate methodology is needed to solve the problem of cost and time due to the experimental approach and the problem of non-spatial property due to the modeling method.

With the development of computational science, the computational fluid dynamics (CFD) has been applied to many industrial fields to solve experimental limitations and cost problems [11-13]. The CFD can be used to predict the flow behavior in ventilated indoor and calculating the concentration of pollutant by solving the partial differential equations with $3 \mathrm{D}$ tensor based on the physics law [14]. In general, the dynamic behavior of particle/aerosol have been predicted with discrete particle method (DPM) and a volume-tracking represented by the volume of fluid (VOF) method in CFD [23-27]. By adopting a numerical method based on the VOF method, various phase flow behaviors, gas-liquid and gas-gas interactions can be accurately described [25-27]. The DPM has been used to track the dynamic behavior of solid particle in air [23,24]. The micro-size liquid aerosols by consumer product spray are volatized into solid phase rapidly [1]. So, the DPM is suitable for application in simulation of spray dynamic behavior. Also, it is possible to analyze the various information such as the dynamic behavior of aerosols that cannot be obtained in the experiment and the ventilation rate of each measurement location [15].

However, despite the various applicability of CFD that can solve the spatial problem of zero dimensional spray models and time/cost problems of experimental method, there are no examples of CFD used in the field of exposure science for estimating the exposure by biocide spray. Therefore, this study proposes the feasibility of applying CFD for the inhalation exposure evaluation by comparing the experiment results and the zero-dimensional simulation results with CFD results. This study has three purposes, (1) to investigate the advantages and disadvantages for the zerodimensional model based on the CFD results and experiment results of the biocide aerosol concentration, (2) to evaluate the feasibility of new approach as the inhalation exposure assessment for solve the spatial problem of zero-dimensional spray model and the cost/time problem of experiment by comparing the CFD results of the aerosol concentration at the various measurement positions with the experiment results of the biocide aerosol concentration at the same measurement positions. As far as we can tell, this paper is first report to propose the new approach of exposure assessment by using CFD. 
Experiment method
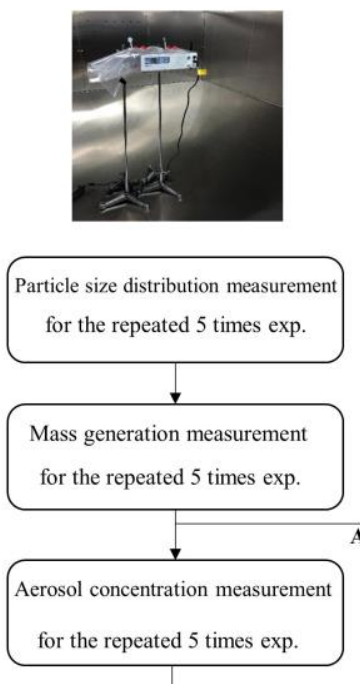

Computational fluid dynamic method

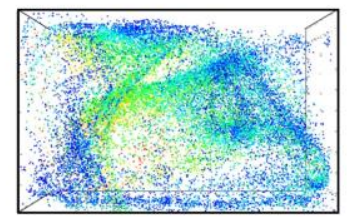

CFD validation for mesh pre-test and continuous phase simulation
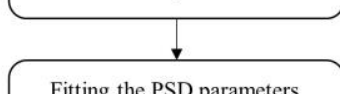

based on experiment data.
Zero-dimensional spray model method

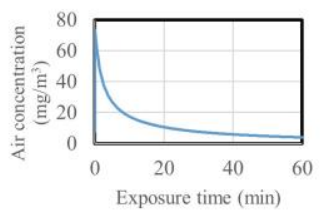

Exposure time (min)

Spray model setup based on Ordinary differential equation

Spray model parameters setting

based on measured exp. data Aerosol size distribution parameters

Aerosol concentration calculation using zero dimensional spray model

Feasibility evaluation of CFD approach for inhalation exposure assessment

Figure 1. Research flow chart.

The research flow chart of this work is summarized as shown Figure 1. First, the experiments such as the particle size distribution (PSD) and mass generation rate measurement are conducted to simulate the zero-dimensional model and CFD. The experiments of the model parameters are performed in a well-controlled large chamber. Then, zero-dimensional spray simulation, CFD spray simulation and the aerosol concentration measurement by spray are performed. The three results were performed based on the same chamber volume and particle information (PSD, mass generation rate and injection time). Finally, the feasibility of zero-dimensional spray model result is evaluated by the CFD results and the measurement results. Also, the CFD results were compared with the experiment results of the biocide aerosol concentration at the same measurement positions in order to verify the feasible use of CFD to exposure estimation.

\section{Methods}

\subsection{Zero-dimesional spray model}

The zero-dimensional spray model simulation was performed to assess the applicability of CFD for the inhalation exposure assessment of biocide spray. The zero-dimensional spray model have been developed to predict the biocide concentration results from the consumer spray products. The spray model was developed considering the effect of ventilation and the force balance acting on particle by the aerosol diameter size. The spray model [4] is as follow:

$$
\begin{gathered}
\frac{d A_{\text {air }}}{d t}=-q_{\text {vent }} A_{\text {air }}(\delta)-v_{s}(\delta) S \frac{A_{\text {air }}}{V}+R_{\text {release }} f_{\text {airborne }} P(\delta, 0) d \delta \\
\frac{d A_{\text {air }}(\delta)}{d t}=-q_{\text {vent }} A_{\text {air }}(\delta)-v_{s}(\delta) S \frac{A_{\text {air }}}{V} \\
C_{\text {air }}(t)=\frac{1}{V} \int A_{\text {air }}(\delta, t) d \delta
\end{gathered}
$$


Where $q_{v e n t}$ is ventilation rate, $A_{\text {air }}$ is total mass of sprayed aerosol/particle $[\mathrm{kg}], \delta$ is the aerosol diameter, $S$ is surface area of room for deposition effect, $V$ is room volume, $R_{\text {release }}$ is rate of release of mass in aerosol particles. $f_{\text {airborne }}$ is rate of non-volatile aerosol from volatile aerosol, $P(\delta, 0)$ is the particle mass distribution function at time $t$ determined from the initial distribution $P(\delta, 0)$. The $v_{s}$ is terminal velocity, which is decided by $\delta$. The $V, \delta$ and $R_{\text {release }}$ are called as a input parameters for the zero dimensional simulation. The input parameters are obtained based on the measurement results. The Equation (1), (2) and (3) represents the total mass of sprayed aerosol/particle during spraying, total mass of sprayed aerosol/particle after spraying and the total concentration in room, respectively. The first term of Equation (1) represents the removal mechanism by ventilation. It is assumed that the concentration of non-volatile aerosol is dispersed in instant. The second term of Equation (1) represents the removal mechanism by the force balance effect depending on particle size. As a result, the space concentration is calculated through the mass according to each aerosol diameter. The model represented ordinary differential equation can quickly predict the aerosol concentration in indoor space. The input parameters used in zero-dimensional spray model are classified into aerosol characteristics and spatial characteristics.

In this study, in the case of spatial characteristics, the $30 \mathrm{~m}^{3}$ chamber with controlled air supply was used in the experiment. Air was supplied from 51 inlets with a diameter of $0.03 \mathrm{~m}$. The total air flow rate is $30 \mathrm{~m}^{3}$. The aerosol input parameters of the zero-dimensional model consist of the PSD (particle size distribution), mass generation rate and exposure factor et. al. The PSD is an important input parameter that affects the results of the zero-dimensional spray simulation and CFD simulation because the particle/aerosol behavior depends on particle diameter size. To set the PSD measurement results equally for CFD and zero-dimensional models, the PSD data was converted to Rosin-Rammler curve. The Rosin-Rammler curve can describe the PSD by using the parameters such as the mean aerosol/particle diameter and spread number. The Rosin-Rambler equation is as follow [16]:

$$
Y_{d}=1-e^{-\left(\frac{d}{d}\right)^{n}}
$$

Where $Y_{d}$ is the cumulative mass fraction, bigger than a given particle/aerosol diameter $d, \bar{d}$ is the mean of particle/aerosol diameter and $n$ is spread number. In other words, the PSD was described by the Rosin-Rammler function. The mean diameter of particle/aerosol and spread parameter were determined by curve fitting the experimental results to the Rosin-Rammler function.

The PSD experiment results for aerosol by biocide spray was obtained as the average value by repeating the 5 times measurement using GRIM OPC equipment, which can be measured in 32 channels from $0.1 \mu \mathrm{m}$ to $30 \mu \mathrm{m}$. The Figure 2 (a) represents the mass fraction by the diameter of particle/aerosol. The mass fraction results in the Figure 2 (a) was converted as the cumulative mass fraction curve. The Figure $2(\mathrm{~b})$ represent the experimental and fitted cumulative mass fraction curve. A minimum aerosol diameter size of $0.1 \mu \mathrm{m}, \bar{d}$ size of $15.4 \mu \mathrm{m}$, maximum aerosol diameter size of $30 \mu \mathrm{m}$ and $n$ values of 1.55 were used as the input condition of zero-dimensional spray simulation and CFD simulation. The mass generation rate determines the total aerosol concentration that occurs in the spray experiment. The mass generation rate was measured by the weight before and after dispensing by repeating the 5 times measurements. In addition, various parameters were also set to simulate the zero-dimensional model such as the sprayed time, simulation time and exposure factor. The spray model parameters were organized as Table 1 . The measured PSD and mass generation rate were connected equally to the zero-dimensional model and CFD model. 


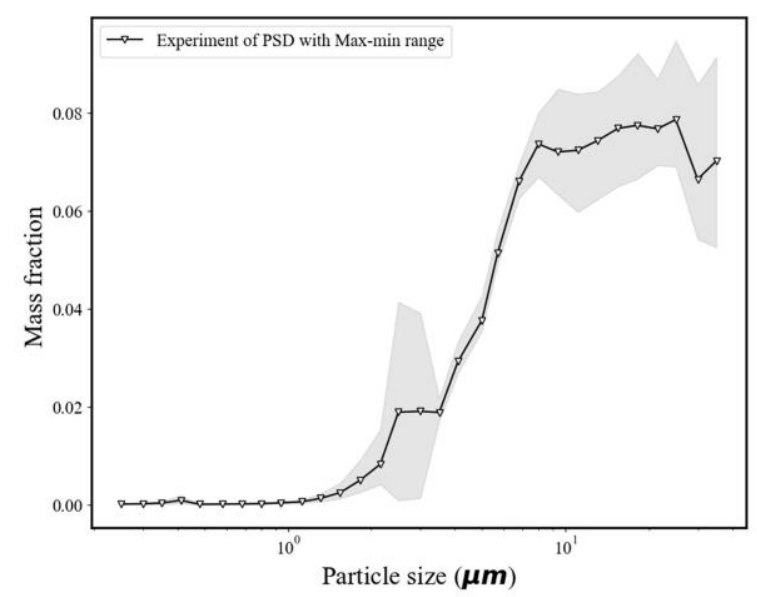

(a)

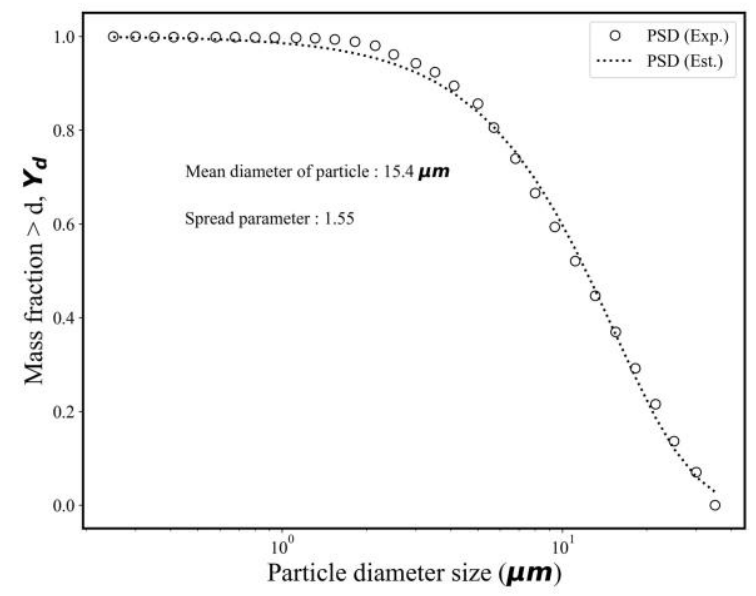

(b)

Figure 2. Results of measurement for mass fraction by aerosol diameter (a) and experimental and fitted cumulative mass fraction curve by aerosol diameter (b). The aerosol size distribution was measured as repeated method. Mean of PSD for aerosol was converted as Rossin-Rambler curve to obtain the aerosol information such as mean of aerosol diameter and spread number.

Table 1 ConsExpo spray model parameters.

\begin{tabular}{cc}
\hline ConsExpo parameters & Values \\
\hline Min/Mean/Max size & $1 \mathrm{e}-07 / 11 \mathrm{e}-06 / 30 \mathrm{e}-06(\mathrm{~m})$ \\
Spread number & 1.5 \\
Mass generation rate & $0.0000119(\mathrm{~kg} / \mathrm{s})$ \\
Weight fraction & 0.007 \\
Density of aerosol & $2\left(\mathrm{~g} / \mathrm{cm}^{3}\right)$ \\
Airborne fraction & 0.6 \\
Injection time & $3 \mathrm{~s}$ \\
Ventilation rate & $1(1 / \mathrm{h})$ \\
Room size & $30 \mathrm{~m}^{3}$ \\
Deposition surface area & $12 \mathrm{~m}^{2}$ \\
\hline
\end{tabular}

\subsection{Measurement of the sprayed biocide concentration}

To evaluate the applicability of CFD for assessment of inhalation exposure by biocide sprays, the concentration of aerosols sprayed in the chamber was measured in the various spot in the chamber. The aerosol concentrations in the chamber were measured at the six position to rationally analyze the results of the zero-dimensional spray model and CFD model. The measurement positions and chamber dimensions were represented as shown Figure 3. The sprayed aerosol concentration was measured repeatedly 5 times in a well-controlled chamber to account for the uncertainty. The measurement lasted during the 60 minute after sprayed aerosol. The concentration of aerosol was obtained by integration of the mass by particle size distribution measured by using the GRIMM OPC equipment. 


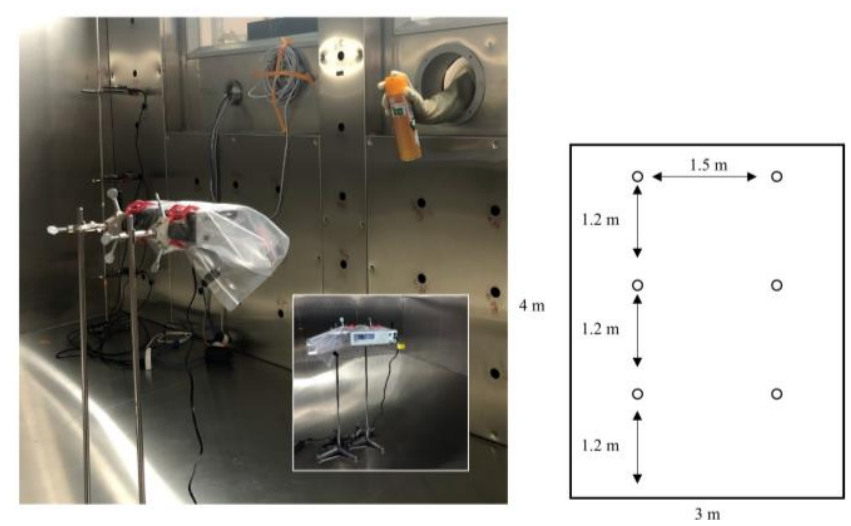

Figure 3. Experiment setup, measurement positions and chamber dimensions. The experiment was performed in ventilation chamber of $30 \mathrm{~m}^{3}$.

\subsection{Numerical simulatiom}

A three-dimensional numerical simulations were performed to evaluate the feasibility of new approach as the inhalation exposure assessment of biocide spray. For estimating the inhalation exposure by biocide spray, $k-\varepsilon-\mathrm{RNG}$ model was used to capture the turbulence flow in indoor chamber, and the discrete phase model (DPM) was used to track the aerosol dynamic behavior. Also, the particle source in cell (PSI-C) algorithm by UDF was adopted for calculating the sprayed aerosol concentration. The numerical simulations were conducted by using the commercial CFD solver (ANSYS FLUENT).

\subsubsection{Modeling the flow field}

The sprayed biocide aerosol has the micro-size diameter. The micro-size aerosol is dependent on the flow field [20]. In the indoor air flow, a complex three-dimensional turbulent flow is generated. Therefore, it is important to capture the turbulence flow in indoor in order to investigate the biocide aerosol behavior. For numerical simulation of turbulent behavior, the 3-D Reynolds average NavierStokes (RANS) equation solved based on finite volume method. The RANS equation is as follow:

$$
\begin{gathered}
\frac{\partial \rho}{\partial t}+\frac{\rho \partial \bar{u}_{i}}{\partial x_{j}}=0 \\
\frac{\partial \bar{u}_{i}}{\partial t}+\bar{u}_{j} \frac{\partial \bar{u}_{i}}{\partial x_{j}}=-\frac{1}{\rho} \frac{\partial \bar{p}}{\partial x_{i}}+\frac{\partial^{2} \bar{u}_{i}}{\partial x_{j} \partial x_{j}}-\frac{\partial}{\partial x_{j}} \tau_{i j}
\end{gathered}
$$

where the Reynolds stress tensor $\tau_{i j}$ can be defined as:

$$
\tau_{i j}=\overline{u_{i}^{\prime} u_{j}{ }^{\prime}}
$$

Here, the $\bar{u}_{i}$ and $u_{i}^{\prime}$ are the mean velocity and fluctuating velocity, respectively. $\bar{p}$ is pressure of fluid. The Reynolds stress tensor due to turbulence behavior are solved by adopting the turbulence models such as $k-\varepsilon, k-\varepsilon-$ RNG and RSM etc. Since the flow field is different depending on the turbulence models, an appropriate turbulence model should be used $[18,19]$.

It has been proven that the $k-\varepsilon, k-\varepsilon-\mathrm{RNG}$ that assumes the Boussinesq eddy viscosity can well capture the flow circulating in indoor chamber relatively better than the RSM model. The $k-$ $\varepsilon-$ RNG was show slightly better results that $k-\varepsilon$ model [14]. The RSM assuming the Reynolds stress tensor with three-dimensional anisotropic property predicted strong circulating flow. Therefore, in this work, we use the $k-\varepsilon-$ RNG model for evaluating the feasibility of CFD approach 
for the inhalation exposure assessment in this study. The equation of $k-\varepsilon-$ RNG model is well described in the following references $[16,17]$.

\subsubsection{Modeling the aerosol motion}

The biocide aerosol motion in indoor chamber was investigated by Eulerian-Lagrangia approach, which consider the air flow as a continuous phase, and the aerosols as a dispersed phase. The sprayed biocide aerosol is transported in continuous phase. The sprayed aerosols were sufficiently diluted that they did not affect the air flow, and particle-to-particle interactions were not considered in aerosol behavior. Also, the aerosols were assumed as the non-volatile particles. The micro-size liquid aerosols by consumer product spray are volatized into solid phase rapidly [2]. Therefore, the DPM is suitable for application in simulation of spray dynamic behavior. To simulate the sprayed biocide aerosol dynamic behavior, the discrete phase model (DPM) are used [16]. the particle tracking equation is as follow:

$$
\frac{d \vec{u}_{p}}{d t}=F_{D}\left(\vec{u}_{k}+u_{k}^{\prime}-\vec{u}_{p}\right)+\frac{\vec{g}\left(\rho_{p}-\rho\right)}{\rho_{p}}+F
$$

Where $\overrightarrow{\mathrm{u}}_{\mathrm{p}}$ is the particle velocity, $\overrightarrow{\mathrm{u}}_{\mathrm{k}}$ is the fluid velocity, $\mathrm{u}_{\mathrm{k}}^{\prime}$ is the turbulent velocity fluctuation, $\rho_{\mathrm{p}}$ is the density of particle, $\rho$ is the density of fluid, the term $F_{D}$ is the drag force per unit particle mass, and $F$ is an additional acceleration (force/unit particle mass).

Although real turbulence flows apply random movements to particles, the RANS model cannot solve all small turbulence flow due to the averaged effect. The particle trajectory is affected by turbulent velocity fluctuations. The probabilistic tracking method is used to deal with the turbulent velocity fluctuation [18]. The probabilistic tracking method was used to accurately predict the particle behavior with DPM [19]. In this work, the DPM with stochastic particle tracking method was used. The description on the governing equations of DPM are detailed in the following reference $[18,19]$ due to the repeated explanation.

\subsubsection{Modeling the aerosol concentration}

The DPM with Lagranian method does not provide functionality about the calculation of aerosol concentration. The particle source in cell (PSI-C) algorithm based on UDF of FLUENT was applied to calculate the aerosol concentration in the specific location. The PSI-C method was developed with the DPM variables. The PSI-C is as follow:

$$
C_{j}=\frac{\dot{M} \sum_{i=1}^{m} d t_{(i, j)}}{V_{i}}
$$

Where $C$ is the average aerosol concentration in a cell, $\dot{M}$ represents the mass flow rate, the subscript $(i, j)$ means the $i^{t h}$ tracking particle and the $j^{t h}$ cell, respectively, $d t$ is the aerosol resident time, $V$ is the cell volume. The PSI-C code is represented as shown Figure 4. 


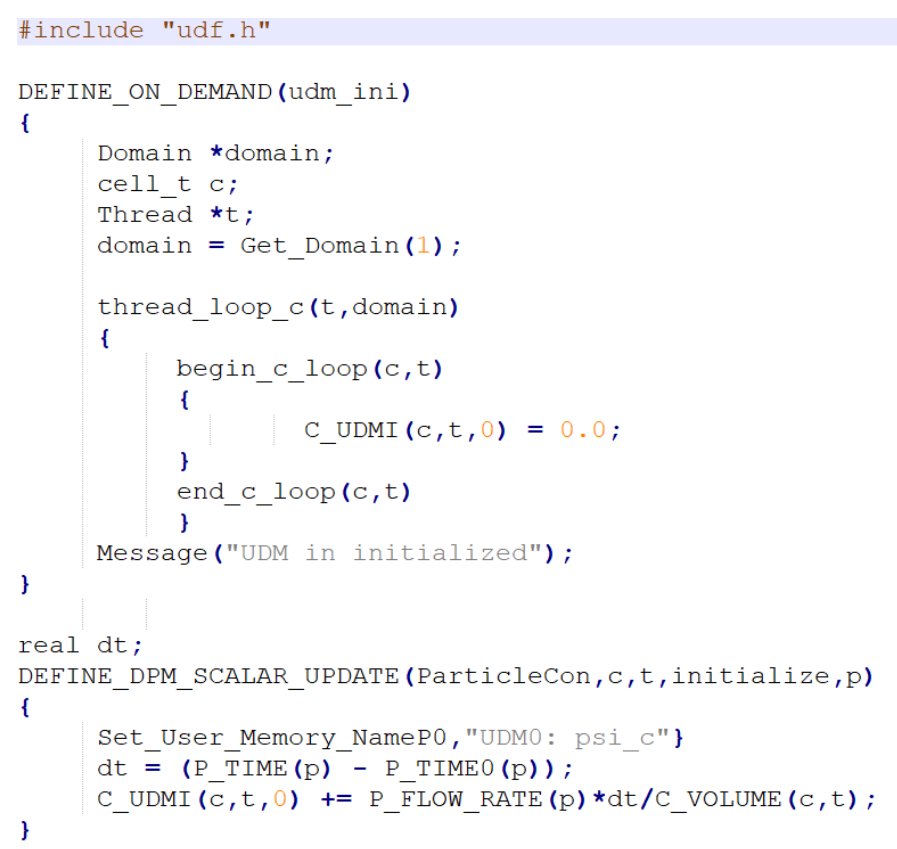

Figure 4. PSI-C UDF code.

\subsubsection{Numerical setting and Domain}

Before evaluating the applicability of CFD for assessment of inhalation exposure by biocide spray, two preliminary tests were conducted to set the suitable simulation condition. The first is the solution convergence test about the aerosol dynamic behavior simulation and the second is the grid test.

In the solution convergence test of the first preliminary test, the residuals of the mass, momentum and turbulence equation did not decrease below $10^{-3}$ when transient analysis was used. So, as a solution convergence strategy, in the first step, we used a steady-state solution using the kepsilon model as an initial guess to provide the solution stability. And then, transient analysis was performed using the $k-\varepsilon-\mathrm{RNG}$ with a second-order upward discretization method. It was confirmed that the residuals of the mass, momentum, and turbulence equations were reduced to less than $10^{-5}$ by the solution convergence strategy. Up to ten iterations were used to converge each time step. The SIMPLE algorithm, PRESTO! and Second order upwind scheme were used for pressure term, pressure-velocity term and turbulence kinetic and dissipation and momentum term, respectively. The criteria of residual values of the turbulence equation and mass/momentum equation for assessing CFD convergence were set as $10^{-5}$ and $10^{-5}$.

For the grid-dependent test of the second preliminary test, the measurement results of a velocity distribution in an indoor chamber were cited by considering time/cost. The grid type is the hexahedron type. The near-wall treatment was achieved by using the scalable wall functions considering the grid refinement with $\mathrm{y}^{+}<11$. The grid independence test was conducted by comparing the velocity profile CFD results of three type mesh (coarse, moderate and fine) with the velocity profile measurement results [14] to derive the optimal grid size. The optimized grid size is applied to the simulation conditions of this work for the sprayed biocide spray simulation. The total number and mesh size of coarse types are $3.5 \times 10^{5}$ and $0.05 \mathrm{~m}$, respectively. The total number and mesh size of moderate types are $2.8 \times 10^{6}$ and $0.015 \mathrm{~m}$, respectively. The total number and mesh size of fine types are $7.5 \times 10^{6}$ and $0.007 \mathrm{~m}$, respectively. The Figure 4 represented the result of the grid dependence test. As a result of comparing the velocity profile measured at three locations $0.2 \mathrm{~m}, 0.4$ $m$, and $0.6 m$ from the wall with simulated results by changing the grid size, it can be seen that the chamber flow can be efficiently predicted when $0.015 \mathrm{~m}$ of maximum length of grid is used. The mesh size has the reasonable computational cost and high accuracy. Therefore, in this study, the maximum length of grid was set to $0.015 \mathrm{~m}$. 

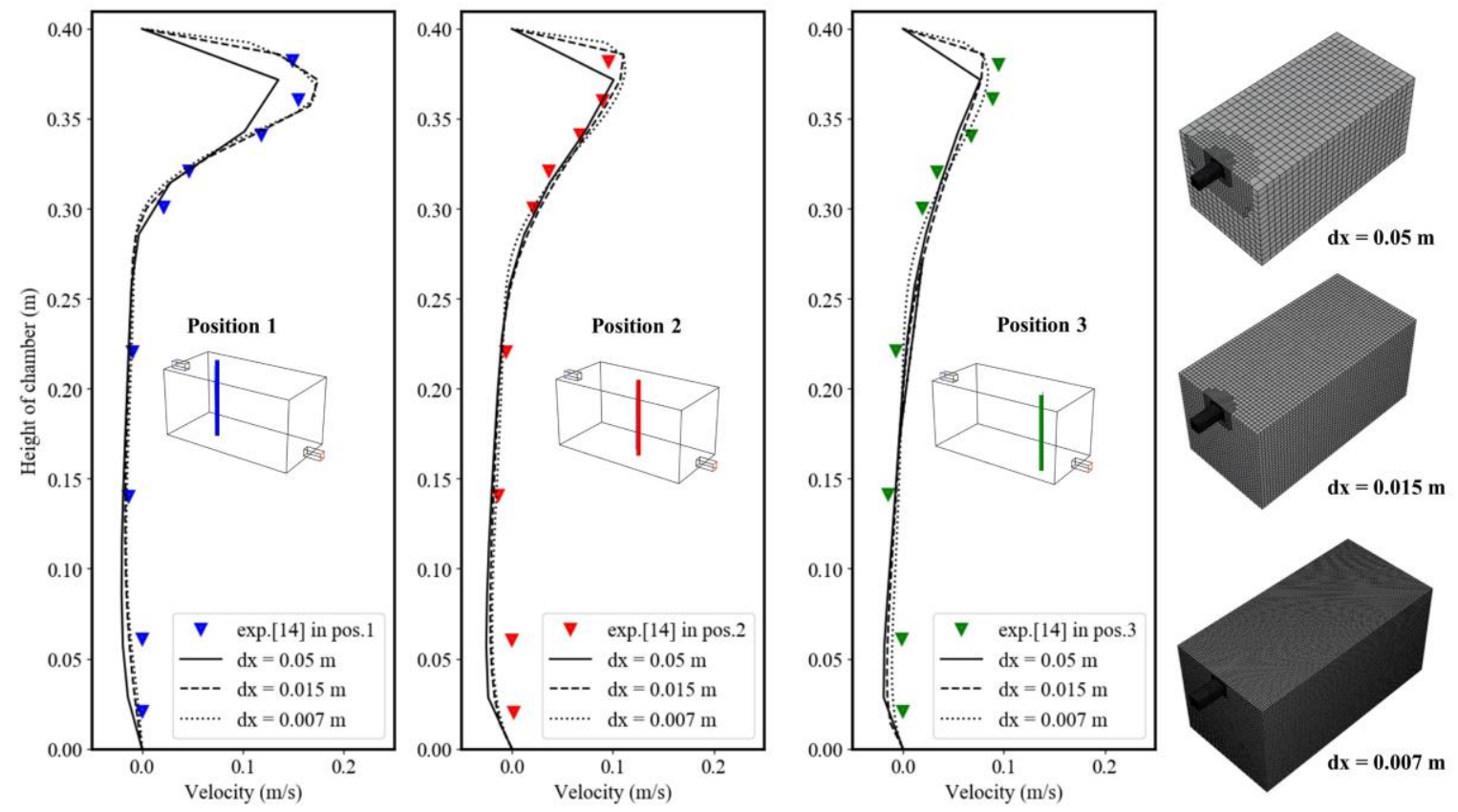

Figure 5. Mesh independent results. The experiment data was cited [14]. The max length of grid is decreased as $0.05,0.015$ and $0.007 \mathrm{~m}$. The measurement positions are $0.2,0.4$ and $0.5 \mathrm{~m}$ from wall.

So, based on preliminary tests results, in this work, $k-\varepsilon-$ RNG model was used to capture the turbulence flow in indoor chamber, and the discrete phase model (DPM) was used to track the aerosol dynamic behavior. Also, the particle source in cell (PSI-C) algorithm by UDF with DPM variables was adopted for calculating the sprayed aerosol concentration. The three modeling method can be applied to estimate the inhalation exposure by biocide spray.

\subsubsection{Numerical method for exposure assessment of aerosol concentration by biocide spray}

We investigated the feasibility of applying CFD for evaluation of inhalation exposure to solve the spatial problem of zero dimensional spray models and the time/cost problem of experimental method. First, we investigated the underestimation possibility of exposure estimation by zerodimensional spray model based on CFD and measurement results. Then, the CFD results of the sprayed aerosol concentration in various position was compared by actual measurement results in order to investigate the CFD applicability for the inhalation exposure assessment of the sprayed biocide aerosol considering the time/cost problem of experimental method.

The measurement position, boundary condition and computation mesh domain of room chamber used in this study represents as shown the Figure 6. The computation mesh was generated as hexahedron type. The green box was called the near field with $1 \mathrm{~m}^{3}$. The other space zone is called as the far field. The zones were used to investigate the possibility of underestimation of exposure estimation by spatial problem of zero dimensional spray model. The aerosol was sprayed in the center of near field for 3 seconds. The aerosol parameter such as PSD, mass generation rate was used in the obtained measurement results in session 2.3.1. As aforementioned solution stability strategy, the continuous phase was first simulated for 2000 iteration in steady-state. Then, the transient simulation was proceeded with DPM combined PSC-I method for 3600 seconds. The micro-size liquid aerosols by consumer product spray are volatized into solid phase rapidly [2]. Therefore, the DPM is suitable for application in simulation of spray dynamic behavior. The CFD setup for used model, boundary condition and DPM condition in this study was summarized in Table 2 and Table 3. 


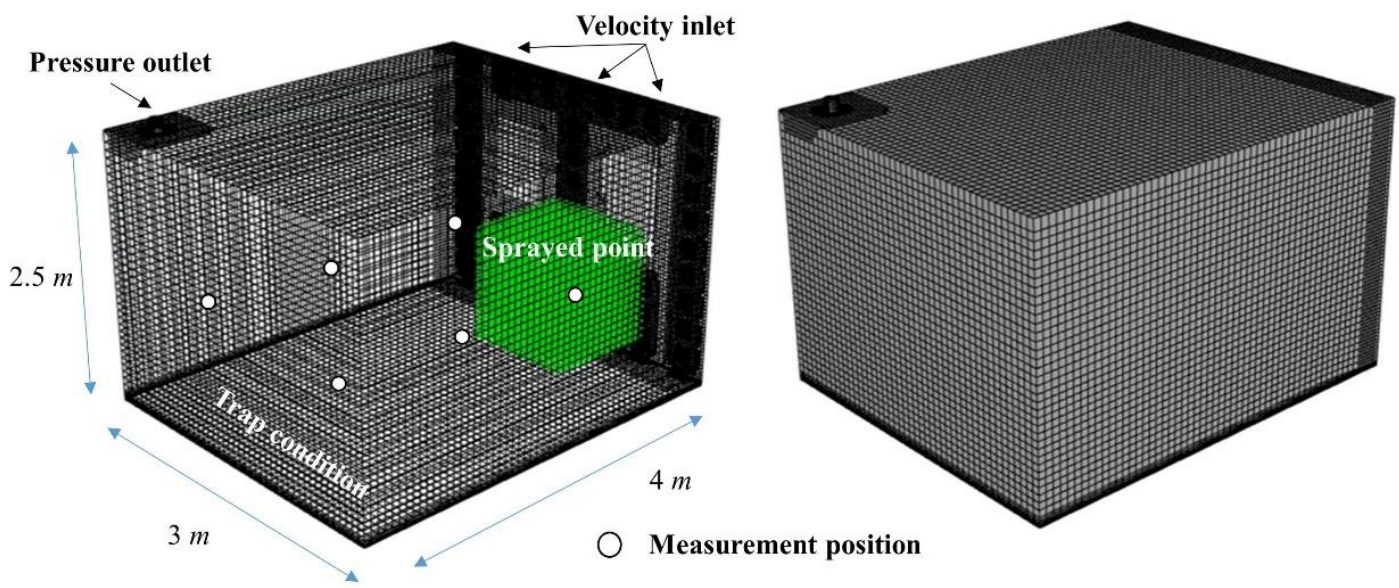

Figure 6. The measurement position, boundary condition and computation mesh domain of room chamber. The computational domain is divided into near field (green) and far field for validating the zero-dimensional spray model.

Table 2. CFD parameter for simulation

\begin{tabular}{cc}
\hline Boundary condition & Values \\
\hline Inlet condition (inlet velocity) & $30 \mathrm{~m}^{3} / \mathrm{h}$ \\
Outlet condition (pressure drop) & $1(\mathrm{~atm})$ \\
Time step size & $0.1(\mathrm{sec})$. \\
Number of time step & 36000 \\
Room size & $30 \mathrm{~m}^{3}$ \\
Number of cell & 435,620 \\
Maximum length of grid & $0.015 \mathrm{~m}$ \\
\hline
\end{tabular}

Table 3. DPM parameter for simulation

\begin{tabular}{cc}
\hline DPM condition & Values \\
\hline Aerosol size range (Min/Max) & 0.1 to $30(\mu \mathrm{m})$ \\
Aerosol spread number & 1.5 \\
Mass generation rate & $0.0000119(\mathrm{~kg} / \mathrm{s})$ \\
A Number of aerosols & 500,000 \\
Density of aerosol & $2\left(\mathrm{~g} / \mathrm{cm}^{3}\right)$ \\
Injection time & $3(\mathrm{sec})$. \\
Injection type & Spray solid cone \\
Aerosol Injection condition & $0.5(\mathrm{kPa})$ \\
\hline
\end{tabular}

\section{Results}


3.1. Evaluation on the underestimate possiblity of inhaluation exposure by zero-dimensional spray model based on CFD and experiment results.

The possibility of underestimation by the zero-dimensional spray model was confirmed in two steps. The first step was to evaluate the zero-dimensional spray model while verifying the validity of the CFD analysis based on the experimental results. In order to reflect the momentary aerosol spread assumption of the zero-dimensional spray model, the aerosol concentration over the total volume of space (far field) was calculated by using CFD, and the experiment results at six locations were averaged (far field).

In the second step, in order to analyze the possibility of underestimation, the simulated concentration value and the experimental value at a specific location (near field) near the spray were compared with the zero-dimensional spray model results. The aerosol concentration of CFD was calculated over time by using the $k-\varepsilon-$ RNG model, PSI-C method, DPM model and the pre-tested simulation conditions. The CFD simulation time is the same as the experiment and zero-dimensional spray model simulation time. The results by three methods were compared for 3600 seconds of physic/simulation. The aerosol by spray was injected for 3 second in three methods. The aerosol concentration of zero dimensional spray model was calculated by solving the ODE for spray behavior.
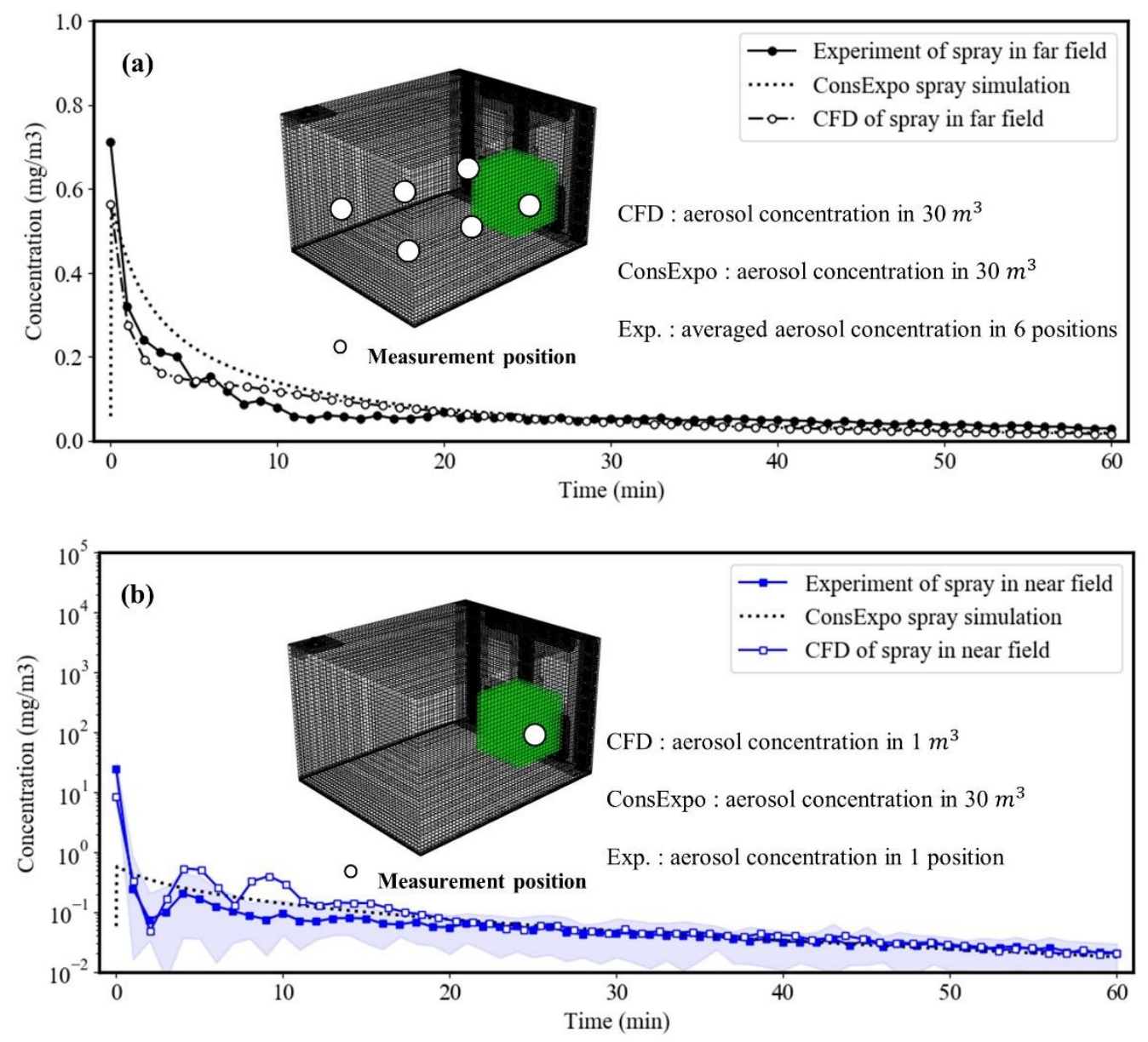

Figure 7. The comparison results of experiment results, zero-dimensional spray simulation (ConsExpo simulation) and CFD simulation in near field and far field. (a) Far field comparison results, (b) Near field - far field comparison results.

The Figure 7 (a) showed the comparison results for three methods in the far field. As shown in the Figure 7 (a), the spray model results, the experiment results in far field and CFD in far field show relatively good results for each other. After spraying, the aerosol concentration decreases rapidly 
within 5 minutes because the large diameter particles quickly deposits to the bottom. This phenomenon can be confirmed through the aerosol dynamic behavior result of 5 minutes by CFD method in Figure 8 (a). After 5 minutes, aerosol of micro diameter throughout the space are diluted to the entire space and removed to the upper outlet by ventilation. When the aerosol is sufficiently diluted, it can be seen that aerosols with the small diameters are circulated throughout the space depending on the flow velocity field in the Figure 8 (b).

Particle diameter
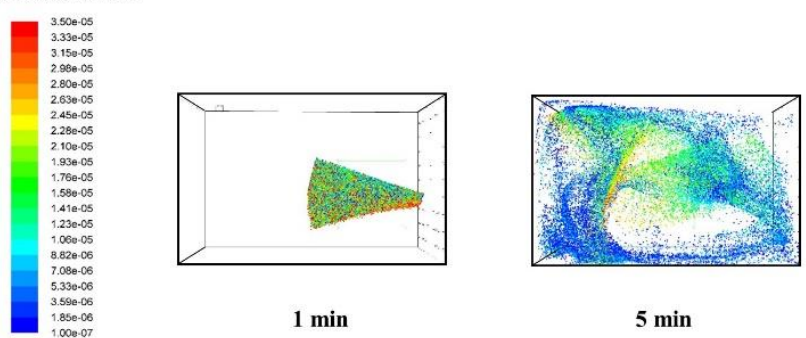

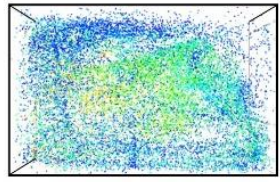

$10 \mathrm{~min}$

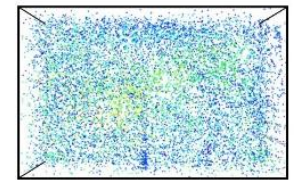

$20 \mathrm{~min}$

(a)

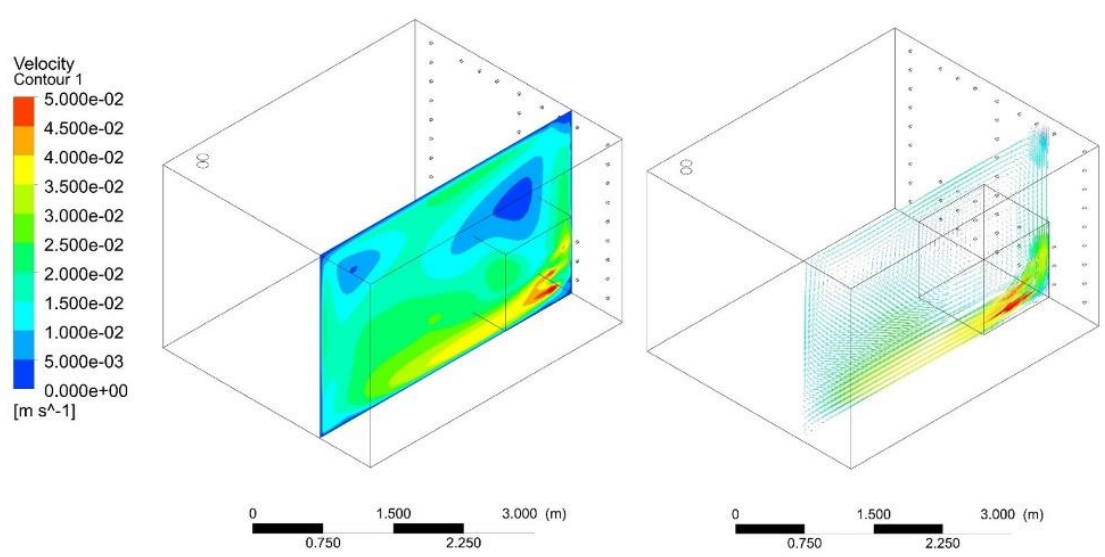

(b)

Figure 8. The dynamic behavior of sprayed biocide aerosol over simulation time (a). Visualization of velocity contour and vector in chamber (b). The dynamic behavior of sub-micro aerosol is dependent to turbulence flow.

Figure 7 (b) represents the result of comparing the CFD result of aerosol concentration and the aerosol concentration measurement result in the near field with the result of the zero-dimensional spray model. The Figure 7 (b) showed that the CFD aerosol concentration results at a specific location and the experimental aerosol concentration values differ from that of the zero dimensional spray model. This is a phenomenon caused by aerosol propagation as shown Figure 8 (a). The CFD results in near field show similarity to the mean-max experimental results in near field over time. That is, the zero-dimensional spray model that ignores spatial characteristics can predict the results contrary to the actual exposure evaluation depending on the location, and CFD method can well explain the aerosol propagation caused by the actual turbulence.

In addition, Table 3 showed the exposure concentration of time-weight average by the spray model, CFD modeling and experimental method. The spray model shows approximately 5 times underestimated when compared with the CFD results in near field. We showed the CFD results of exposure in near field is similar to the measurement result in near field quantitatively. Therefore, it can be seen that CFD and experimental methodologies taking into account spatial characteristics are needed for proper inhalation exposure assessment.

Table 4. Aerosol concentration by time-average for experiment results in near field and far field, CFD results in near field and far field and ConsExpo simulation. 
Unit : $\mathrm{mg} / \mathrm{m}^{3} \quad \sim 10 \mathrm{~min} \quad \sim 20 \mathrm{~min} \quad \sim 30 \mathrm{~min} \quad \sim 40 \mathrm{~min} \quad \sim 50 \mathrm{~min} \quad \sim 60 \mathrm{~min}$

Experiment results (near field) field)

CFD results (near field)

CFD results (far field)

ConsExpo model results
Experiment results (far
1.12

0.22

0.24
1.3

0.14

0.11

0.63

0.44

0.16

0.13

0.28

0.24

0.17

0.13

0.11

0.09

0.08

\subsection{Evaluation on the feasibility of CFD for inhaluation exposure assessment based on experiment results.}

The measurement results of the concentration of aerosols at five locations and the CFD results at the same five locations were compared for solving the experimental problem of time/cost and for evaluating the possibility of inhalation exposure at various locations by using CFD. The 5 measurement are a positions other than the near field. The measurement data where the aerosol concentration rises highest at each measurement location were compared with the CFD results of the same time and location as shown Figure 9.

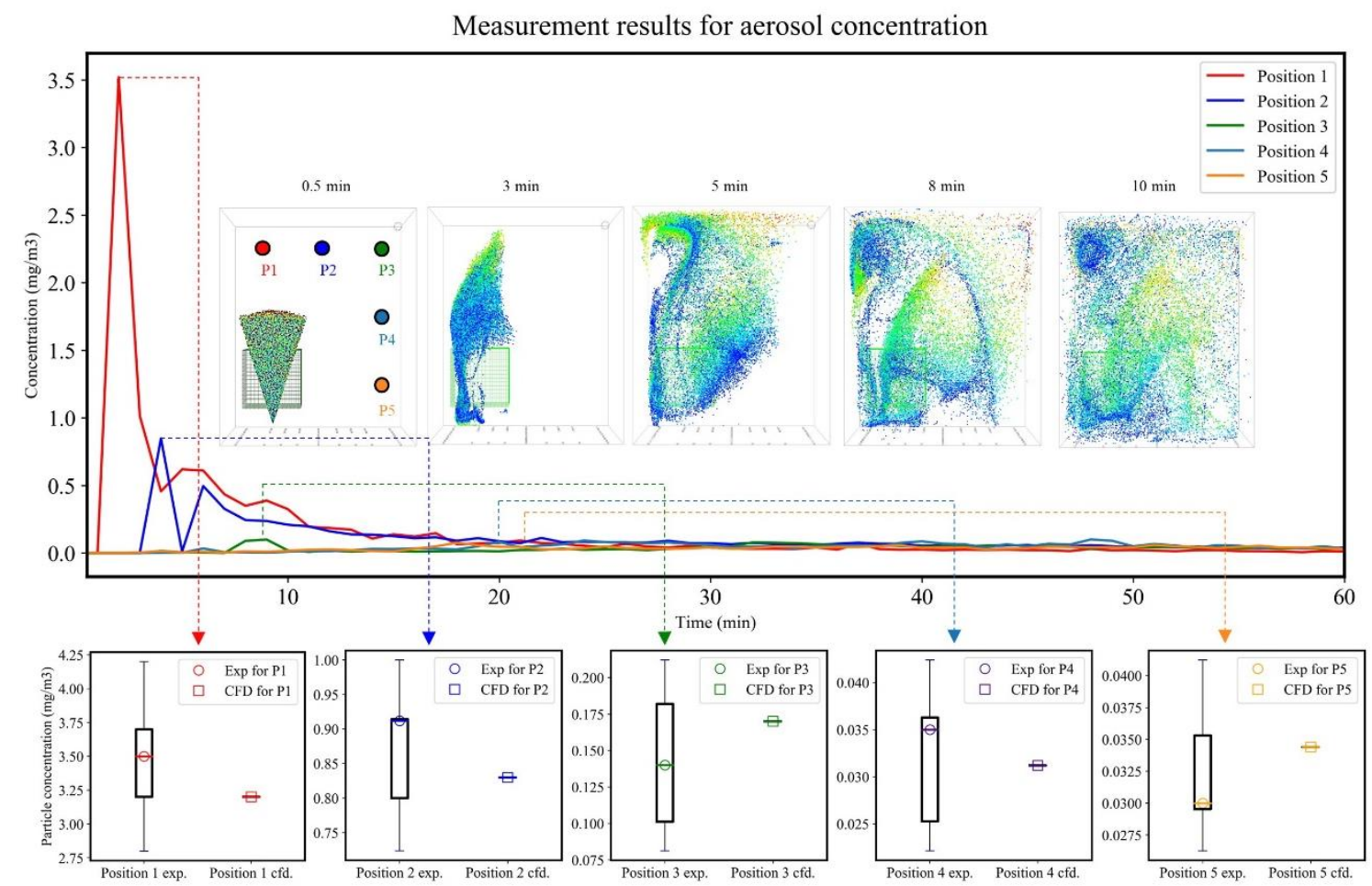

Figure 9. Comparison of measurement result with CFD results in the 5 measurement position. The error bar is represented due to repeated experiment. The circle is the average measurement results, which is the peak concentration. The CFD results according to the measurement position is similar with the measurement results. 
The aerosol concentration by measurement position were calculated due to the aerosol propagation. The experiment was independently performed 5 times. The experimental result is an error bar representing the minimum, mean and maximum with standard deviation. The CFD value at each measurement location is within the experimental error range. It means the CFD condition such as mesh size, numerical schemes and modeling methods are set correctly. That showed the concentration of aerosol exposure at various locations can be reasonably evaluated through CFD. It means that the CFD approach can be successfully applied to exposure estimation.

\section{Conclusion}

In this paper, we investigated the applicability of CFD for the inhalation exposure assessment of biocide spray in order to solve the spatial problem of zero dimensional space model and the time/cost-consuming problem of measurement method. The CFD results of the sprayed aerosol concentration in various position was compared by measurement results and the zero-dimensional spray model results. The results by three methods were compared for 3600 seconds of physic/simulation. The conclusion can be drawn as follow:

(1) The zero dimensional spray model results, the experiment results in far field and CFD in far field show relatively good results for each other. However, the spray model shows approximately 5 times underestimated when compared with the CFD results in near field and experiment in near field. In this study, it was shown that exposure evaluation should be performed using CFD or experimental methods rather than conventional zero dimensional spray for human health.

(2) When the concentration measurement is performed at various locations using experimental methodology, the cost and time required are enormously consumed. the results of measuring the concentration of aerosols at five locations and the CFD results at the same location were compared to show the possibility of evaluating inhalation exposure at various locations using CFD. The experimental result is an error bar representing the minimum, average and maximum. It can be seen that the CFD value at each measurement location is within the experimental error range. In this study, it was shown that the experimental cost and time problem for inhalation exposure can be reasonably solved using CFD.

In other words, in the field of exposure science, a guideline for exposure evaluation using CFD was found that complements the shortcomings of the conventional methodology, the zerodimensional spray model and measurement method.

However, the CFD requires a relatively the high computational cost for simulation of exposure estimation. It is difficult to estimate the exposure to the user's fast needs. To address these compute cost problems, machine learning modeling method based on simulation data are used in the various industries [20-22]. Therefore, in future research, we will develop a machine learning model with spatial information as input variable and spatial concentration as output variable to predict concentration according to various spatial structure and characteristics.

Author Contributions: D.P. analyzed the numerical data and organized draft paper.; J.H.L. performed the experiment. All authors have read and agreed to the published version of the manuscript.

Funding: This research was supported by Korea Environment Industry \& Technology Institute (KEITI) through Environmental Health Action Program, funded by Korea Ministry of Environment (MOE) (No. 2012001370007, NTIS 1485012429

\section{Acknowledgments: none}

Conflicts of Interest: The authors declare no potential conflict of interest with respect to the research, authorship and publication of this article.

\section{References}

1. RIVM. ConsExpo nano tool. www.consexponano.nl. www.consexpoweb.nl (2007, October 30)

2. RIVM. Consexpo Web. www.consexpoweb.nl (2016, October 30). 
3. US EPA. Consumer exposure model (CEM) user guide. www.epa.gov/sites/production/files/201906/documents/cem_2. 1_user_guide.pdf. (2019, December 11)

4. Delmaar, J. E., \& Bremmer, H. J., Modeling and experimental validation of the inhalation exposure of consumers to aerosols from spray cans and trigger sprays (Report No. 320104005/2009). RIVM. 2009.

5. Young, B. M., Tulve, N. S., Egeghy, P. P., Driver, J. H., Zartarian, V. G., Johnston, J. E., Barnekow, D. E. Comparison of four probabilistic models (CARES ${ }^{\circ}$, CalendexTM, Zero-dimensional, and SHEDS) to estimate aggregate residential exposures to pesticides. Journal of Exposure Science and Environmental Epidemiology. 2012, 22(5), 522-532. https://doi.org/10.1038/jes.2012.54

6. Arnold, S. F., \& Ramachandran, G. Influence of parameter values and variances and algorithm architecture in ConsExpo model on modeled exposures. Journal of Occupational and Environmental Hygiene. 2014, 11(1), 54-66. https://doi.org/10.1080/15459624.2013.816430

7. Delmaar, C., \& Meesters, J. Modeling consumer exposure to spray products: an evaluation of the ConsexpoWeb and Consexponano models with experimental data. Journal of Exposure Science \& Environmental Epidemiology. 2020, 30, 878-887. doi:10.1016/j.powtec.2017.11.012.

8. Park, J., Yoon, C., \& Lee, K. Comparison of modeled estimates of inhalation exposure to aerosols during use of consumer spray products. International Journal of Hygiene and Environmental Health. 2018, 221(6), 941-950. https://doi.org/10.1016/j.ijheh.2018.05.005

9. Kwon, K.S.; Lee, I.B.; Han, H.T.; Shin, C.Y.; Hwang, H.S.; Hong, S.W.; Bitog, J.P.; Seo, I.H.; Han, C.P. Analysing ventilation efficiency in a test chamber using age-of-air concept and CFD technology. Biosyst. Eng. 2011, 110, 421-433, doi:10.1016/j.biosystemseng.2011.08.013.

10. Cherrie, J. W., MacCalman, L., Fransman, W., Tielemans, E., Tischer, M., \& Van Tongeren, M. Revisiting the effect of room size and general ventilation on the relationship between near- and far-field air concentrations. Annals of Occupational Hygiene. 2011, 55(9), 1006-1015. https://doi.org/10.1093/annhyg/mer092

11. Park, D., Cha, J., Kim, M., \& Go, J. S. Multi-objective optimization and comparison of surrogate models for separation performances of cyclone separator based on CFD, RSM, GMDH-neural network, back propagation-ANN and genetic algorithm. Engineering Applications of Computational Fluid Mechanics. 2020, 14(1), 180-201. https://doi.org/10.1080/19942060.2019.1691054

12. Cha, J., Kim, M., Park, D., \& Go, J. S. Experimental determination of the viscoelastic parameters of K-BKZ model and the influence of temperature field on the thickness distribution of ABS thermoforming. International Journal of Advanced Manufacturing Technology. 2019, 103(1-4), 985-995. https://doi.org/10.1007/s00170-019-03408-8

13. Liu, Z., Li, A., Xu, X., \& Gao, R. Computational fluid dynamics simulation of airflow patterns and particle deposition characteristics in children upper respiratory tracts. Engineering Applications of Computational Fluid Mechanics. 2012, 6(4), 556-571. https://doi.org/10.1080/19942060.2012.11015442

14. Chen, F., Yu, S. C. M., \& Lai, A. C. K. Modeling particle distribution and deposition in indoor environments with a new drift-flux model. Atmospheric Environment. 2006, 40(2), 357-367. https://doi.org/10.1016/j.atmosenv.2005.09.044

15. Zhou, Y., Deng, Y., Wu, P., \& Cao, S. J. The effects of ventilation and floor heating systems on the dispersion and deposition of fine particles in an enclosed environment. Building and Environment. 2017, 125, 192-205. https://doi.org/10.1016/j.buildenv.2017.08.049

16. ANSYS Inc.ANSYS FLUENT Theory Guide; ANSYS FLUENT-16.1; ANSYS Inc.: Canonsburg, PA, USA, 2018.

17. Adedoyin, A. A., Walters, D. K., \& Bhushan, S. Investigation of turbulence model and numerical scheme combinations for practical finite-volume large eddy simulations. Engineering Applications of Computational Fluid Mechanics. 2015, 9(1), 324-342. https://doi.org/10.1080/19942060.2015.1028151

18. Rahimzadeh, H., Maghsoodi, R., Sarkardeh, H., \& Tavakkol, S. Simulating flow over circular spillways by using different turbulence models. Engineering Applications of Computational Fluid Mechanics. 2012, 6(1), 100-109. https://doi.org/10.1080/19942060.2012.11015406

19. Tang, Y., Guo, B., \& Ranjan, D. Numerical simulation of aerosol deposition from turbulent flows using three-dimensional RANS and les turbulence models. Engineering Applications of Computational Fluid Mechanics. 2015, 9(1), 174-186. https://doi.org/10.1080/19942060.2015.1004818

20. Park, D.; Go, J.S. Design of cyclone separator critical diameter model based on machine learning and cfd. Processes 2020, 8, 1-13, doi:10.3390/pr8111521. 
21. Yang, C.; Kim, Y.; Ryu, S.; Gu, G.X. Prediction of composite microstructure stress-strain curves using convolutional neural networks. Mater. Des. 2020, 189, 108509, doi:10.1016/j.matdes.2020.108509.

22. Kim, Y.; Yang, C.; Kim, Y.; Gu, G.X.; Ryu, S. Designing an Adhesive Pillar Shape with Deep Learning-Based Optimization. ACS Appl. Mater. Interfaces 2020, 12, 24458-24465, doi:10.1021/acsami.0c04123.

23. He, X.; Xu, H.; Li, W.; Sheng, D. An improved VOF-DEM model for soil-water interaction with particle size scaling. Comput. Geotech. 2020, 128, 103818, doi:10.1016/j.compgeo.2020.103818.

24. Xu, L.; Zhou, X.; Li, J.; Hu, Y.; Qi, H.; Wen, W.; Du, K.; Ma, Y.; Yu, Y. Numerical simulations of molten breakup behaviors of a de laval-type nozzle, and the effects of atomization parameters on particle size distribution. Processes 2020, 8, doi:10.3390/pr8091027.

25. Silva, M.C.F.; Campos, J.B.L.M.; Miranda, J.M.; Araújo, J.D.P. Numerical study of single taylor bubble movement through a microchannel using different CFD packages. Processes 2020, 8, 1-19, doi:10.3390/pr8111418.

26. McCraney, J.; Weislogel, M.; Steen, P. OpenFOAM Simulations of Late Stage Container Draining in Microgravity. Fluids 2020, 5, 207, doi:10.3390/fluids5040207.

27. Tembely, M.; Alameri, W.S.; Alsumaiti, A.M.; Jouini, M.S. Pore-scale modeling of the effect of wettability on two-phase flow properties for newtonian and non-newtonian fluids. Polymers (Basel). 2020, 12, 1-22, doi:10.3390/polym12122832. 